\title{
Transparency of Semi-Insulating, n-Type, and p-Type Ammonothermal GaN Substrates in the Near-Infrared, Mid-Infrared, and THz Spectral Range
}

\author{
Robert Kucharski ${ }^{1}$, Łukasz Janicki ${ }^{2}$, Marcin Zajac ${ }^{1}$, Monika Welna ${ }^{2}$, Marcin Motyka ${ }^{2}$, \\ Czesław Skierbiszewski ${ }^{3}$ and Robert Kudrawiec ${ }^{2, *}$ \\ 1 AMMONO S. A, Prusa 2, 00-493 Warsaw, Poland; Kucharski@ammono.com (R.K.); \\ zajac@ammono.com (M.Z.) \\ 2 Department of Experimental Physics, Wroclaw University of Science and Technology, \\ Wybrzeże Wyspiańskiego 27, 50-370 Wrocław, Poland; lukasz.janicki@pwr.edu.pl (Ł.J.); \\ monika.welna@pwr.edu.pl (M.W.); marcin.motyka@pwr.edu.pl (M.M.) \\ 3 Institute of High Pressure Physics, Polish Academy of Science, Sokołowska 29/37, 01-142 Warsaw, Poland; \\ czeslaw@mail.unipress.waw.pl \\ * Correspondence: robert.kudrawiec@pwr.edu.pl; Tel.: +48-71-320-4280
}

Received: 10 May 2017; Accepted: 21 June 2017; Published: 25 June 2017

\begin{abstract}
GaN substrates grown by the ammonothermal method are analyzed by Fast Fourier Transformation Spectroscopy in order to study the impact of doping (both n- and p-type) on their transparency in the near-infrared, mid-infrared, and terahertz spectral range. It is shown that the introduction of dopants causes a decrease in transparency of GaN substrates in a broad spectral range which is attributed to absorption on free carriers (n-type samples) or dopant ionization (p-type samples). In the mid-infrared the transparency cut-off, which for a semi-insulating GaN is at $\sim 7 \mu \mathrm{m}$ due to an absorption on a second harmonic of optical phonons, shifts towards shorter wavelengths due to an absorption on free carriers up to $\sim 1 \mu \mathrm{m}$ at $\mathrm{n} \sim 10^{20} \mathrm{~cm}^{-3}$ doping level. Moreover, a semi-insulating GaN crystal shows good transparency in the 1-10 THz range, while for n-and p-type crystal, the transparency in this spectral region is significantly quenched below $1 \%$. In addition, it is shown that in the visible spectral region n-type GaN substrates with a carrier concentration below $10^{18} \mathrm{~cm}^{-3}$ are highly transparent with the absorption coefficient below $3 \mathrm{~cm}^{-1}$ at $450 \mathrm{~nm}$, a satisfactory condition for light emitting diodes and laser diodes operating in this spectral range.
\end{abstract}

Keywords: GaN; infrared spectral range; $\mathrm{THz}$ spectral range; free-carrier absorption

\section{Introduction}

A choice of growth substrate for III-N based technology is directed most often by the cost effectiveness of the solution combined with a final quality of the device. Sapphire substrates and $\mathrm{GaN} /$ sapphire templates are commonly chosen, but their applicability is often limited by low thermal conductivity of sapphire $\left(0.25-0.50 \mathrm{~W} / \mathrm{cm} \mathrm{K}\right.$ at $100{ }^{\circ} \mathrm{C}$ [1]). This can be avoided if the growth is performed on a $\mathrm{SiC}$ substrate which has superior thermal properties to sapphire (thermal conductivity of $5 \mathrm{~W} / \mathrm{cm} \mathrm{K}$ at $100{ }^{\circ} \mathrm{C}$ ) [2]. However, a higher cost and larger lattice mismatch [3] often prevents this solution. Ideally, III-N devices should be grown on a native substrate such as $\mathrm{GaN}$ which can be grown by a hydride vapor phase epitaxy or the ammonothermal method. The first of those methods offers a low impurity level but suffers from wafer bow. Ammonothermal GaN boasts the highest crystalline quality in the field but unintentional doping can be a problem [4]. Work is being done in order to combine the best qualities of both methods $[5,6]$. 
Ammonothermal bulk GaN crystals can be grown with an intended conductivity level by controlling the unintentional oxygen content during the growth process, leading to an electron concentration up to $\sim 10^{20} \mathrm{~cm}^{-3}$ (n-type) and an intentional $\mathrm{Mg}$ concentration up to $\sim 10^{19} \mathrm{~cm}^{-3}$ (p-type) [7]. The ammonothermal method can also obtain semi-insulating (SI) GaN crystals which are crucial for power electronics [8] via appropriate compensation of oxygen donors by $\mathrm{Mg}$ acceptors. While the possibility of obtaining conductive substrates is beneficial in some areas, the impact of introduced dopants on the transparency of the crystals can be detrimental in others. Such examples are GaN-based intersubband devices which operate in a mid-infrared spectral range $[9,10]$ and thereby need substrates which are transparent in this spectral range. On the other hand, a high doping can achieve a significant refractive index contrast between the substrate and the device stack. Such doped substrates (i.e., plasmonic substrates) have been shown as effective bottom cladding for mode guiding in InGaN/GaN optoelectronic devices, including lasers operating in a visible spectral range [11,12].

Semi-insulating (SI) GaN is known to be transparent from $0.36 \mu \mathrm{m}$ to $\sim 7 \mu \mathrm{m}$ where an absorption on a second harmonic of optical phonons occurs [13-15]. This broad transparency window allows fabrication of optical devices which operate outside the typical spectral range (e.g., at $1.55 \mu \mathrm{m}$ ) related with III-N structures (i.e., structures which emit UV, blue, and green light). In order to achieve emission at such low energies, intersubband transitions are used. Several devices operating on this principle have already been proposed $[9,10]$. However, several factors can influence the transparency of GaN in the infrared range. It has been shown that $\mathrm{GaN}$ grown on sapphire and $\mathrm{SiC}$ (i.e., GaN/sapphire and $\mathrm{GaN} / \mathrm{SiC}$ templates) has a narrower transparency window [14]. The use of additional substrates (in this case sapphire and $\mathrm{SiC}$ ) leads to a shift of the long wavelength cut-off due to non-transparency of these substrates. Another, rather well known, mechanism leading to absorption in the infrared region is the introduction of free carriers. An introduction of $10^{19} \mathrm{~cm}^{-3}$ electrons by $\mathrm{O}$ doping shifts the cut-off from $\sim 7 \mu \mathrm{m}$ to well below $2 \mu \mathrm{m}$ [14]. There is, however, no systematic study showing the dependency of the cut-off position on the carrier concentration including $\mathrm{n}$ - and $\mathrm{p}$-type doping. In [14], only semi-insulating and highly doped $\left(\mathrm{n} \sim 10^{19} \mathrm{~cm}^{-3}\right)$ bulk GaN substrates were compared to unintentionally doped $\mathrm{GaN}$ layers grown on sapphire and $\mathrm{SiC}$.

In the 7-14 $\mu \mathrm{m}$ window (excluding the narrow 9-10 $\mu \mathrm{m}$ ) GaN is not transparent due to an absorption on the Restrahlen band [13]. However, at longer wavelengths, the radiation should be able to penetrate the material. It is especially interesting to study this in the context of growing interest in terahertz imaging. III-N based devices have been shown to be suitable for detection and emission of terahertz waves. Devices based on an intraband absorption in quantum wells [16,17] or plasma wave excitation [18] have been presented. However, the transparency of GaN crystals in the terahertz range has not been studied yet.

While the transparency window of SI GaN is $0.36-7 \mu \mathrm{m}$, doping can significantly affect it not only in the infrared and at longer wavelengths, but also in the visible range. This is unfavorable from the optoelectronics point of view where a reabsorption of the light emitted from an active part of the device (i.e., a quantum well) can occur in the doped passive part (e.g., a contact layer). In this way, a significant part of the emission may be blocked.

In this paper we analyze the optical transparency of SI and doped ammonothermal GaN substrates in the near-infrared, mid-infrared, and terahertz spectral range. In particular, we analyze the effect of doping on the blueshift of the cut-off wavelength in the mid-infrared region. Semi-insulating substrates and n-type doped crystals with doping concentration in the range from $4.4 \times 10^{17} \mathrm{~cm}^{-3}$ to $9 \times 10^{19} \mathrm{~cm}^{-3}$ are examined significantly extending the work done in [14]. The influence of p-type doping on the cut-off wavelength shift is also analyzed. In the terahertz range, the loss of transparency due to the doping is studied. In addition, we show how the absorption coefficient is affected by the carrier concentration in the visible and near-infrared region where $\mathrm{GaN}$ crystals are quite transparent. 


\section{Materials and Methods}

Bulk GaN crystals for this study were grown by a basic ammonothermal method [7,8]. The doping was introduced by a controlled introduction of dopants $(\mathrm{O}$ and $\mathrm{Mg})$ into the growth autoclave. Crystals were cut into $0.5 \mathrm{~mm}$ thick slices and polished on both sides for transparency measurements.

Transmission measurements were performed at room temperature on a Bruker Vertex $80 \mathrm{v}$ customized Fourier spectrometer [19]. In the near- to mid-infrared spectral range a Globar was used as a light source and a mercury-cadmium-telluride photoconductor as a detector. In the terahertz range, a bolometric detection was used with an optical cut off $\left(700 \mathrm{~cm}^{-1}\right)$ filter and the same Globar lamp as a source.

Absorption measurements were performed using a Cary5 (Varian) spectrophotometer operating in the UV-VIS-NIR range $(250-3300 \mathrm{~nm})$ at room temperature. All the spectra were corrected for multiple reflections of light from the GaN sample. The absorption coefficient was calculated using the following formula, which assumes a normal light incidence

$$
T=\frac{(1-R)^{2} e^{-\alpha d}}{1-R^{2} e^{-2 \alpha d}}
$$

where $T$-measured transmission, $d$-sample thickness, $\alpha$-absorption coefficient, and $R$-measured reflection. Both transmission and reflection were measured independently for each sample.

To determine electrical properties (carrier concentration) of the studied samples, both impedance spectroscopy and Hall measurements have been applied. For these purposes, an impedance analyzer Agilent 4294A and a MMR Technologies Hall system were used.

\section{Results and Discussion}

Figure 1 shows 1 inch low n-type highly transparent GaN, 2 inches n-type $\left(n=10^{19} \mathrm{~cm}^{-3}\right) \mathrm{GaN}$ and 1.5-inch SI GaN substrates of polar orientation produced by the Ammono company. They can be prepared in an epiready standard for both Ga- and N-polar sides and their conductivity can be controlled from n-type to p-type. Such 2 inch and 1 inch substrates are widely used for fabrication of AlGaN/GaN transistors (SI substrates) and InGaN/GaN lasers (n-type substrates). As seen with the naked eye, they are very well transparent in the visible spectral range. However, the transparency of $\mathrm{GaN}$ is changing with the increase in the doping concentration and in the visible spectral range it can be seen with the naked eye, see Figure 2 which shows n-type and p-type GaN samples with various doping concentrations and the change of color due to the doping. These ammonothermal GaN crystals are the subject of studies of transparency in the near-infrared, mid-infrared, and $\mathrm{THz}$ spectral range. In addition, for n-type substrates, which are widely used in light emitters operating in the visible spectral range, the absorption coefficient is determined in this spectral range.

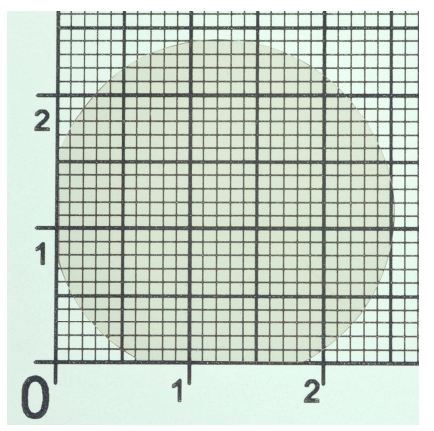

(a)

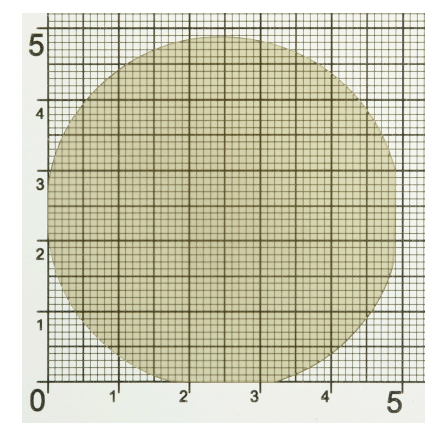

(b)

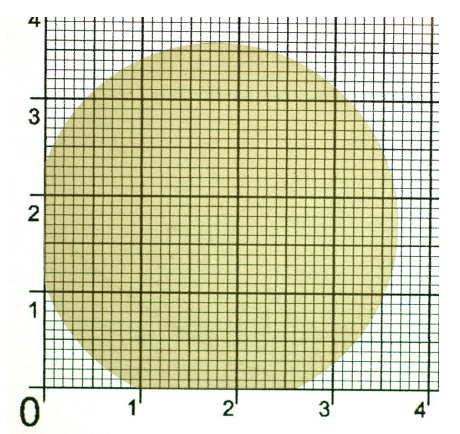

(c)

Figure 1. GaN substrates obtained by the ammonothermal method: (a) 1" low n-type highly transparent, (b) 2" n-type $\left(10^{19} \mathrm{~cm}^{-3}\right)$, and (c) $1.5^{\prime \prime}$ SI-type. 


\section{O-doped GaN:}

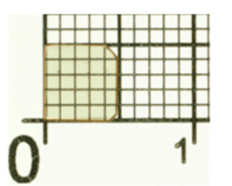

$4.4^{*} 10^{17} \mathrm{~cm}^{-3}$



$3.0^{*} 10^{18} \mathrm{~cm}^{-3}$



$1.3^{*} 10^{19} \mathrm{~cm}^{-3}$

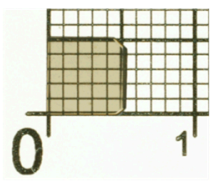

$5.6^{*} 10^{19} \mathrm{~cm}^{-3}$

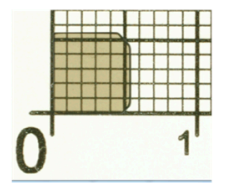

$9.0^{*} 10^{19} \mathrm{~cm}^{-3}$

\section{Mg-doped GaN:}



SI



SI

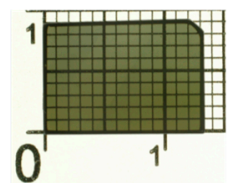

$1.0^{*} 10^{18} \mathrm{~cm}^{-3}$

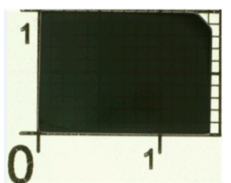

$5.0^{*} 10^{18} \mathrm{~cm}^{-3}$



$1.0^{*} 10^{19} \mathrm{~cm}^{-3}$

Figure 2. $\mathrm{O}$ (top row) and $\mathrm{Mg}$ (bottom row) doped GaN crystals of various carrier concentrations.

Figure 3a shows transmission spectra measured at room temperature for $\mathrm{GaN}$ crystals doped by oxygen with various concentrations of free electrons. A spectrum recorded for a SI GaN is included as a reference. A gradual decrease of transparency is visible with an increase in free carrier concentration and the cut-off wavelength shifts towards shorter wavelengths. To illustrate the influence of the carrier concentration on the transparency wavelengths corresponding to $30 \%$ and $10 \%$ transparency are plotted in Figure 3b. The cut-off wavelength shifts linearly towards the visible range on a semi-logarithmic plot. This effect is attributed to absorption on free carriers as all dopants are ionized at room temperature as schematically shown in the sketch in Figure 3b. This feature could be used to tune the transparency of $\mathrm{GaN}$ in the $1-7 \mu \mathrm{m}$ spectral range.

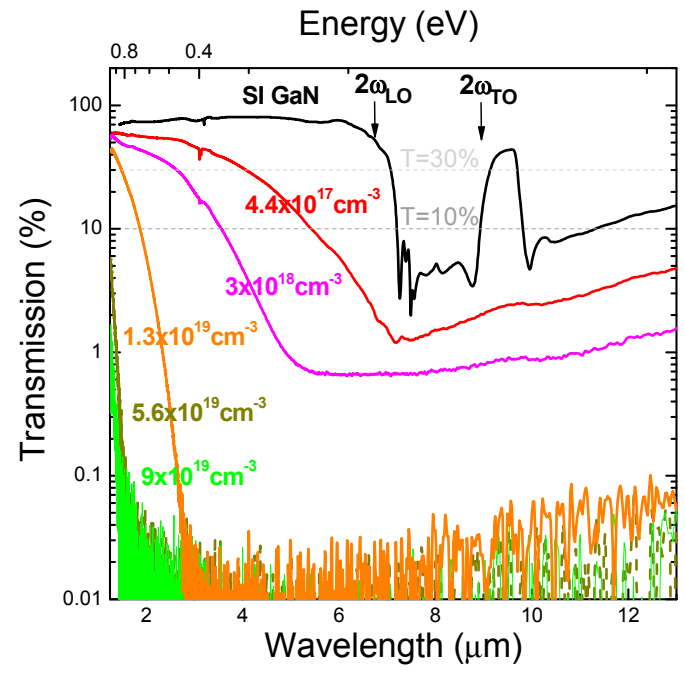

(a)

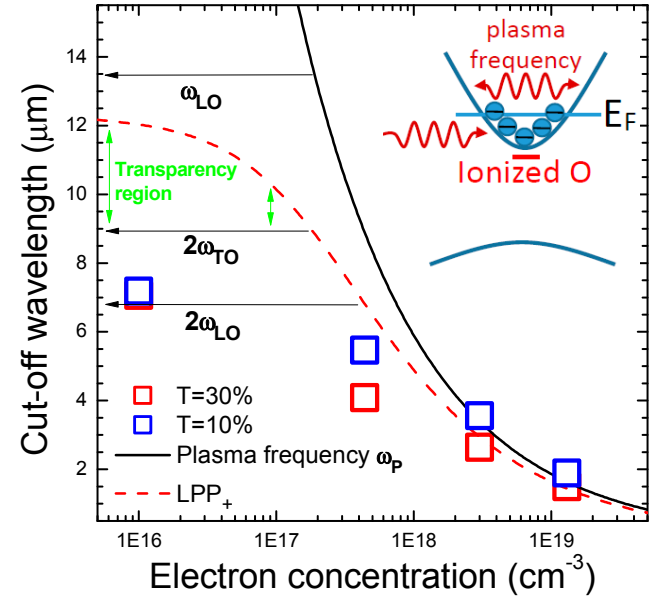

(b)

Figure 3. (a) Transmission spectra of n-type GaN of various electron concentrations (color lines) together with a transmission spectrum of SI GaN measured at room temperature in the near- and mid-infrared spectral region. (b) The transparency cut-off wavelength (open squares) for n-type GaN of various electron concentrations taken at the transparency of 10\% (blue points) and 30\% (red points) together with curves representing plasma frequency and high energy branch of longitudinal phonon-plasmon $\left(\mathrm{LPP}_{+}\right)$. In the sketch $\mathrm{E}_{\mathrm{F}}$ means the Fermi level. 
In general, the free carrier absorption is a well-known phenomenon in semiconductors which has been also observed for n-type GaN [13-15,20-22]. In order to compare our experimental results with a quantitative analysis, the plasma frequency $\left(\omega_{P}\right)$ has been calculated for various carrier concentrations according to Equation (2)

$$
\omega_{P}^{2}=\frac{n e^{2}}{m^{*} \varepsilon_{\infty} \varepsilon_{0}}
$$

where $n$ and $e$ are the free electron concentration and the elementary charge, respectively, $m^{*}$ is the effective electron mass which is assumed to be $0.22 m_{0}$ ( $m_{0}$ is the electron rest mass) [23], $\varepsilon_{\infty}$ is the high frequency limit of the dielectric constant assumed 5.35, and $\varepsilon_{0}$ is the vacuum dielectric constant. The calculated plasma frequency is plotted in Figure $3 \mathrm{~b}$ by a solid black line. In addition, the mode of high energy branch of longitudinal phonon-plasmon $\left(\mathrm{LPP}_{+}\right)$is plotted by a dashed red line in Figure $3 \mathrm{~b}$. This mode is calculated according to the following formula

$$
\omega_{L P P_{+}}^{2}=\frac{\omega_{L O}^{2}+\omega_{P}^{2}}{2}+\sqrt{\left(\frac{\omega_{L O}^{2}+\omega_{P}^{2}}{2}\right)^{2}-\omega_{T O}^{2} \omega_{P}^{2}}
$$

where $\omega_{L O}$ and $\omega_{T O}$ are the zero carrier density frequencies of the $E_{1}$ longitudinal (LO) and transversal optical (TO) phonon frequencies [14].

For a low electron concentration $\left(n<10^{17} \mathrm{~cm}^{-3}\right)$, the transparency of a GaN substrate in the mid-infrared region ends at $\sim 6.8 \mu \mathrm{m}$, which is labeled as $2 \omega_{L O}$ in Figure 3 and corresponds to the second harmonic $\mathrm{LO}$ phonon generation in GaN. The strong absorption ends at the energy corresponding to two TO phonons which is labeled as $2 \omega_{T O}$ in Figure 3. It is worth noting that, for zinc-blended semiconductors, such as GaAs or InP, the second-harmonic absorption is negligibly weak. In the case of $\mathrm{GaN}$, this absorption is very strong since the $E_{1} \mathrm{LO}$ and TO mode is polarized transverse to the $\mathrm{c}$ axis similarly like the incident radiation. Next, a window of sample transparency is observed in the $\sim 9-10 \mu \mathrm{m}$ range and no transparency is observed above $10 \mu \mathrm{m}$ for the SI sample. An absorption due to the first harmonic $\mathrm{LO}$ phonon generation in $\mathrm{GaN}$ is expected at longer wavelengths (the energy of a LO phonon corresponds to $\sim 13.5 \mu \mathrm{m}$ ), but this absorption can be significantly broadened due to phonon-phonon scattering [13] and/or can be attributed to $\mathrm{LPP}_{+}$, see dashed red line in Figure $3 \mathrm{~b}$. In this figure, it is also visible that the transparency window, see green arrow in Figure $3 \mathrm{~b}$ defined between $\mathrm{LPP}_{+}$and $2 \omega_{T O}$, is narrowing with the increase in electron concentration. Above the electron concentration $n>2 \times 10^{17} \mathrm{~cm}^{-3}$ this window reduces to zero. With the next increase in electron concentration, the cut-off for transparency shifts to a shorter wavelength due to a free carrier absorption (black solid line) and the $\mathrm{LPP}_{+}$absorption. These theoretical predictions are very consistent with experimental data which corresponds to $30 \%$ (red open squares) and $10 \%$ (blue open squares) transparency of the sample. In addition, it is visible that the mid-infrared absorption edge becomes sharper with the increase in electron concentration, compare slopes of the absorption edge in Figure 3a or the spectral positon of red and blue squares in Figure 3b. Summarizing this analysis, it is clearly visible that the transparency of n-type GaN substrates in the near and mid infrared region is controlled by the electron concentration and the wavelength cut-off is very well described by the plasma frequency for $n>1 \times 10^{18} \mathrm{~cm}^{-3}$. The scenario is different for p-type GaN. Figure 4a shows transmission spectra measured for $\mathrm{GaN}$ crystals doped with $\mathrm{Mg}$. At $1 \times 10^{18} \mathrm{~cm}^{-3}$ doping level, the transparency curve has a similar shape to that of SI GaN and the cut-off frequency stays at the same position. The notable difference appears at wavelengths $<6 \mu \mathrm{m}$ where a significant drop of transparency is visible. At $\sim 0.2 \mathrm{eV}$ $(6.2 \mu \mathrm{m})$ and higher this drop corresponds to the energy needed to excite electrons from the valence band (VB) to the Mg acceptor level, as schematically shown in the sketch in Figure 3b. An increase in doping concentration to $5 \times 10^{18} \mathrm{~cm}^{-3}$ leads to a strong decrease of the transparency of the whole infrared range. Since the free hole absorption is expected to be negligibly important in this spectral range in these samples due to a low hole concentration and a large effective mass for holes in $\mathrm{GaN}$, this absorption is attributed to optical transitions between (i) the VB and Mg-related acceptors, 
(ii) dopants (Mg-related acceptors and non-intentional donors (both deep and shallow)), (iii) occupied $\mathrm{Mg}$ acceptors and the conduction band (CB), and (iv) the VB and deep donors which are unoccupied by electrons in Mg-doped GaN. These processes are schematically illustrated in Figure $4 \mathrm{~b}$ and their intensities/significance vary with the $\mathrm{Mg}$ concentration in $\mathrm{GaN}$ crystals as well as the concentration of non-intentional donor dopants. A quantitative analysis in this case is difficult, since the model of the defect absorption is not well established for GaN. However, it is possible to estimate the wavelength cut-off which is related to the free hole plasma frequency. For a hole concentration $p=10^{18} \mathrm{~cm}^{-3}$ the cut-off is expected at $\sim 9 \mu \mathrm{m}$, i.e., at much longer wavelengths than this for which no transparency is observed for GaN:Mg samples with $\mathrm{Mg}$ concentration $\geq 5 \times 10^{18} \mathrm{~cm}^{-3}$.

Since the activation energy of $\mathrm{Mg}$ dopants in $\mathrm{GaN}$ is large ( 170 meV [24]), the nature of the transparency of p-type $\mathrm{GaN}$ is different than this one which takes place in n-type GaN where the activation energy of $\mathrm{O}$ donors is comparable with the thermal energy at room temperature (the activation energy of oxygen in $\mathrm{GaN}$ is $\sim 29-32 \mathrm{meV}$ [25-27]). Even if non-intentional deep donors are present in n-type GaN, the free carrier absorption determines the transparency of n-type GaN in the infrared spectral region. In such a case, optical transitions between deep donors and the $C B$ are also expected but their significance is negligible if the electron concentration is high enough, e.g., $>3 \times 10^{18} \mathrm{~cm}^{-3}$ as seen in Figure 3a.



(a)

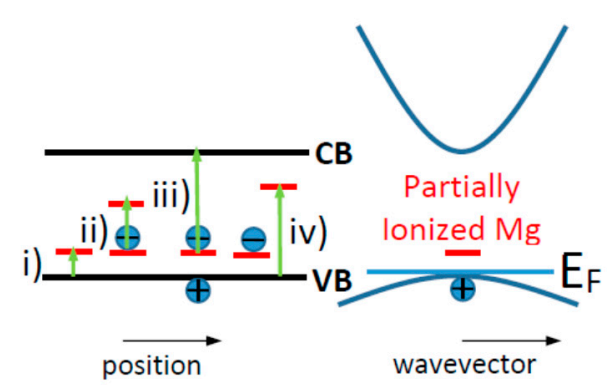

(b)

Figure 4. (a) Transmission spectra of p-type GaN of various hole concentrations (color lines) together with transmission spectra of SI GaN (black and grey lines) measured at room temperature in the nearand mid-infrared spectral region. (b) The sketch of light absorption in p-type GaN which is associated with dopants.

Figure 5 shows transmission spectra measured in the terahertz range (1-14 THz) for SI, n-type with the electron concentration on the level of $10^{18} \mathrm{~cm}^{-3}$, and p-type with the $\mathrm{Mg}$ doping level of $10^{18} \mathrm{~cm}^{-3} \mathrm{GaN}$ crystals. The SI GaN crystal shows $50 \%$ transparency in $1-8 \mathrm{THz}$ range which then drops to below $1 \%$ at $10 \mathrm{THz}$ and higher. The drop starting at $8 \mathrm{THz}$ corresponds to the activation energy of $\sim 32 \mathrm{meV}$ of $\mathrm{O}_{\mathrm{N}}$ (oxygen substituting nitrogen) defect in GaN which acts as a shallow donor [27]. At room temperature $(26 \mathrm{meV})$, only a part of $\mathrm{O}_{\mathrm{N}}$ donors are thermally activated. Remaining inactivated impurities can absorb radiation to release electrons to the conduction band. Since there is a significant amount of oxygen, even in SI GaN crystals grown by ammonothermal method, such an effect is expected and we attribute the drop in transparency at frequencies $>8 \mathrm{THz}$ to the absorption on oxygen impurities. The result shows that $\mathrm{GaN}$ is a suitable material for applications in $1-8 \mathrm{THz}$ and can be considered for use in $8-14 \mathrm{THz}$ only if the structure is thin (so the absorption is 
kept low) and the GaN-based structure is fabricated on a suitable substrate which is also transparent in the terahertz range. On the other hand, doping makes GaN completely non-transparent in the terahertz range with transmission below $0.1 \%$. This effect is present for both $\mathrm{n}$ - and $\mathrm{p}$-type doping. It is expected that the transparency of $\mathrm{GaN}$ in the $\mathrm{THz}$ spectral range can be tuned via $\mathrm{n}$ - or $\mathrm{p}$-type doping below $10^{18} \mathrm{~cm}^{-3}$ but quantitative analysis of this issue needs samples with suitably low unintentional impurity concentration. Moreover, it is worth noting that $\mathrm{SI} \mathrm{GaN} \mathrm{can} \mathrm{be} \mathrm{obtained} \mathrm{via} \mathrm{co-doping,} \mathrm{i.e.,} \mathrm{O}$ dopants can be compensated by Mg dopants etc. This suggests that the transparency of SI GaN in the $\mathrm{THz}$ spectral range can be also tuned. Such an effect was observed for SI GaN crystals obtained by the ammonothermal method. We believe that further studies of transparency of GaN in the THz spectral range require samples with low non-intentional doping, i.e., below $<10^{17} \mathrm{~cm}^{-3}$.



Figure 5. Transmission spectra of SI (black line), n-type (green line), and p-type (blue line) GaN substrates measured at room temperature in the $\mathrm{THz}$ spectral range.

Since conductive GaN substrates are needed for the fabrication of light emitting diodes and laser diodes emitting in the visible (blue-green) spectral range, it is important to know how small the absorption coefficient is at these wavelengths for n-type GaN substrates typically used for production of laser diodes. Figure 6 shows the absorption coefficient $(\alpha)$ determined for such substrates in the visible and near-infrared region. Below $400 \mathrm{~nm}$, the sharp increase in absorption is observed because of the Urbach tail of density of states in the conduction band (O-related donor states). At $n<1 \times 10^{18} \mathrm{~cm}^{-3}$ carrier concentration the absorption coefficient is as low as $2-3 \mathrm{~cm}^{-1}$ at $450 \mathrm{~nm}$ and stays on this level in the whole 450-1200 nm range. An increase in carrier concentration to $3 \times 10^{19} \mathrm{~cm}^{-3}$ and higher causes $\alpha$ to increase as well. The change is lowest at around $630 \mathrm{~nm}$ and most pronounced in the infrared. While the performance of blue and green laser diodes may be affected by partial absorption of light in doped layers, this influence might be avoided by reducing the thickness of the aforementioned layers. In infrared, however, $\alpha$ reaches a value of $\sim 100 \mathrm{~cm}^{-1}$. This can significantly impede the efficiency of intersubband GaN-based emitters. Suitable steps must be taken in order to avoid this effect.

Transparency of p-type GaN in the visible spectral range is significantly affected by a dopant-related absorption which was previously discussed. This phenomenon is observed with the naked eye in Figure 2 for p-type samples. Therefore, p-type $\mathrm{GaN}$ substrates are less interesting for light emitters operating in blue-green spectral range and the absorption coefficient in visible spectral range is not analyzed in this paper. In general, the sharp decrease in transparency due to doping is a well-known phenomenon for $\mathrm{Mg}$-doped $\mathrm{GaN}$ in light emitters since the top cladding is p-type and can significantly absorb the light emitted from the InGaN/GaN quantum well region. In order to minimize this effect, the thickness of the Mg-doped layer is reduced as much as it is possible and hence the p-type layer 
is more transparent. GaN crystals shown in Figure 2 are $\sim 0.5 \mathrm{~mm}$ thick and therefore transparency of p-type samples with larger Mg concentration is very poor in this case but it improves with the reduction of substrate thickness.

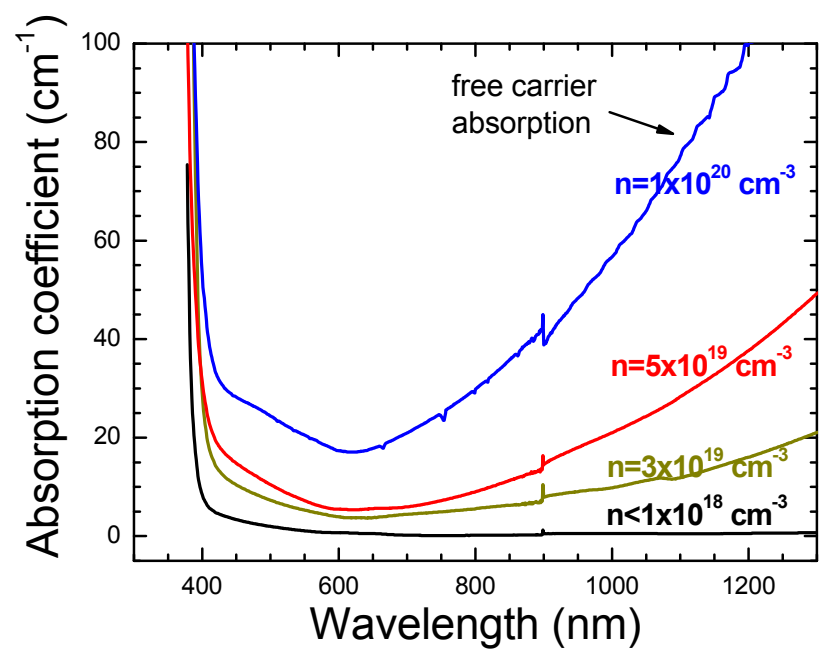

Figure 6. The absorption coefficient determined in the visible and near-infrared spectral region for n-type GaN substrates dedicated for light emitters.

\section{Conclusions}

Transparency measurements of SI, O-, and Mg-doped bulk ammonothermal GaN substrates reveal the strong impact of doping in near-infrared, mid-infrared, and terahertz regions. N-type doping, through the absorption on free carriers, can be employed to tune the transparency of GaN crystals in the mid- to near-infrared by shifting the cut-off wavelength in a controlled manner. On the other hand, p-type doping could potentially reduce the transparency in the near-infrared while keeping it on a high level in the mid-infrared. However, a relatively small change in $\mathrm{Mg}$ concentration can bring the transparency close to zero in the whole infrared range and the mechanism of light absorption in this spectral range is more complex, since it depends on a concentration of $\mathrm{Mg}$ dopants as well as non-intentional donor-like dopants. At the terahertz frequencies, any kind of doping causes a dramatic reduction of the transparency. A semi-insulating GaN can maintain transparency in the $1-8 \mathrm{THz}$ range at a high level. For laser applications in the visible and near-infrared, keeping as low a carrier concentration as possible is beneficial as the absorption coefficient can be kept as low a $2-3 \mathrm{~cm}^{-1}$ in the $450-1200 \mathrm{~nm}$ range.

Acknowledgments: This work was performed within the grant of the National Center for Research and Development (grant PBS3/A3/23/2015).

Author Contributions: Robert Kucharski has grown all these samples, prepared GaN samples for optical studies, and supervised this project at the Ammono company; Lukasz Janicki was responsible for electrical characterization and has prepared the first version of the manuscript; Marcin Zajac controlled doping issues, performed absorption measurements in UV-VIS-NIR range; Monika Welna and Marcin Motyka performed transmission measurements in the near-infrared, mid-infrared, and THz range; Czeslaw Skierbiszewski tested GaN substrates in molecular beam epitaxy, discussed results, supervised this project at Institute of High Pressure Physics, and coordinated the whole PBS project between involved institutions; Robert Kudrawiec analyzed experimental data, revised the manuscript, and supervised the project at Wroclaw University of Science and Technology. All authors corrected the final version of the manuscript.

Conflicts of Interest: The authors declare no conflict of interest.

\section{References}

1. Cooke, H.F. High-Power GaAs FET Amplifiers; Artech House: Norwood, MA, USA, 1993; pp. 227-261. 
2. Morkoç, H.; Strite, S.; Gao, G.B.; Lin, M.E.; Sverdlov, B.; Burns, M. Large-band-gap SiC, III-V nitride, and II-VI ZnSe-based semiconductor device technologies. J. Appl. Phys. 1994, 76, 1363-1398. [CrossRef]

3. Liu, L.; Edgar, J.H. Substrates for gallium nitride epitaxy. Mater. Sci. Eng. R Rep. 2002, 37, 61-127. [CrossRef]

4. Dwiliński, R.; Doradziński, R.; Garczyński, J.; Sierzputowski, L.P.; Puchalski, A.; Kanbara, Y.; Yagi, K.; Minakuchi, H.; Hayashi, H. Excellent crystallinity of truly bulk ammonothermal GaN. J. Cryst. Growth 2008, 310, 3911-3916. [CrossRef]

5. Kucharski, R.; Zajac, M.; Puchalski, A.; Sochacki, T.; Bockowski, M.; Weyher, J.L.; Iwinska, M.; Serafinczuk, J.; Kudrawiec, R.; Siemiatkowski, Z. Ammonothermal growth of GaN crystals on HVPE-GaN seeds prepared with the use of ammonothermal substrates. J. Cryst. Growth 2015, 427, 1-6. [CrossRef]

6. Bockowski, M.; Iwinska, M.; Amilusik, M.; Fijalkowski, M.; Lucznik, B.; Sochacki, T. Challenges and future perspectives in HVPE-GaN growth on ammonothermal GaN seeds. Semicond. Sci. Technol. 2016, 31, 093002. [CrossRef]

7. Dwiliński, R.; Doradziński, R.; Garczyński, J.; Sierzputowski, L.P.; Puchalski, A.; Kanbara, Y.; Yagi, K.; Minakuchi, H.; Hayashi, H. Bulk ammonothermal GaN. J. Cryst. Growth 2009, 311, 3015-3018. [CrossRef]

8. Dwiliński, R.; Doradziński, R.; Sierzputowski, L.; Kucharski, R.; Zając, M. AMMONO-GaN substrates for microwave and RF applications. In Proceedings of the 2012 19th International Conference on Microwaves Radar Wireless Communications, Warsaw, Poland, 21-23 May 2012; Volume 1, pp. 99-101.

9. Kandaswamy, P.K.; Machhadani, H.; Bougerol, C.; Sakr, S.; Tchernycheva, M.; Julien, F.H.; Monroy, E. Midinfrared intersubband absorption in GaN/AlGaN superlattices on Si (111) templates. Appl. Phys. Lett. 2009, 95, 141911. [CrossRef]

10. Tchernycheva, M.; Nevou, L.; Vivien, L.; Julien, F.H.; Kandaswamy, P.K.; Monroy, E.; Vardi, A.; Bahir, G. Intersubband optics in GaN-based nanostructures-physics and applications. Phys. Status Solidi B 2010, 247, 1622-1627. [CrossRef]

11. Cywiński, G.; Kudrawiec, R.; Rzodkiewicz, W.; Kryśko, M.; Litwin-Staszewska, E.; Łucznik, B.; Misiewicz, J.; Skierbiszewski, C. Doping-induced contrast in the refractive index for GaInN/GaN structures at telecommunication wavelengths. Appl. Phys. Express 2009, 2, 111001. [CrossRef]

12. Stańczyk, S.; Czyszanowski, T.; Kafar, A.; Czernecki, R.; Targowski, G.; Leszczyński, M.; Suski, T.; Kucharski, R.; Perlin, P. InGaN laser diodes with reduced AlGaN cladding thickness fabricated on GaN plasmonic substrate. Appl. Phys. Lett. 2013, 102, 151102. [CrossRef]

13. Yang, J.; Brown, G.J.; Dutta, M.; Stroscio, M.A. Photon absorption in the Restrahlen band of thin films of GaN and AlN: Two phonon effects. J. Appl. Phys. 2005, 98, 043517. [CrossRef]

14. Welna, M.; Kudrawiec, R.; Motyka, M.; Kucharski, R.; Zając, M.; Rudziński, M.; Misiewicz, J.; Doradziński, R.; Dwiliński, R. Transparency of GaN substrates in the mid-infrared spectral range. Cryst. Res. Technol. 2012, 47, 347-350. [CrossRef]

15. Frayssinet, E.; Knap, W.; Prystawko, P.; Leszczynski, M.; Grzegory, I.; Suski, T.; Beaumont, B.; Gibart, P. Infrared studies on GaN single crystals and homoepitaxial layers. J. Cryst. Growth 2000, 218, 161-166. [CrossRef]

16. Machhadani, H.; Kotsar, Y.; Sakr, S.; Tchernycheva, M.; Colombelli, R.; Mangeney, J.; Bellet-Amalric, E.; Sarigiannidou, E.; Monroy, E.; Julien, F.H. Terahertz intersubband absorption in GaN/AlGaN step quantum wells. Appl. Phys. Lett. 2010, 97, 191101. [CrossRef]

17. Beeler, M.; Bougerol, C.; Bellet-Amalric, E.; Monroy, E. Pseudo-square AlGaN/GaN quantum wells for terahertz absorption. Appl. Phys. Lett. 2014, 105, 131106. [CrossRef]

18. Fatimy, A.E.; Dyakonova, N.; Meziani, Y.; Otsuji, T.; Knap, W.; Vandenbrouk, S.; Madjour, K.; Théron, D.; Gaquiere, C.; Poisson, M.A.; et al. AlGaN/GaN high electron mobility transistors as a voltage-tunable room temperature terahertz sources. J. Appl. Phys. 2010, 107, 024504. [CrossRef]

19. Möllmann, K.-P.; Vollmer, M. Fourier transform infrared spectroscopy in physics laboratory courses. Eur. J. Phys. 2013, 34, S123. [CrossRef]

20. Ibáñez, J.; Hernández, S.; Alarcón-Lladó, E.; Cuscó, R.; Artús, L.; Novikov, S.V.; Foxon, C.T.; Calleja, E. Far-infrared transmission in GaN, AlN, and AlGaN thin films grown by molecular beam epitaxy. J. Appl. Phys. 2008, 104, 033544. [CrossRef]

21. Feneberg, M.; Osterburg, S.; Lange, K.; Lidig, Ch.; Garke, B.; Goldhahn, R.; Richter, R.E.; Netzel, C.; Neumann, M.D.; Esser, N.; et al. Band gap renormalization and Burstein-Moss effect in silicon- and germanium-doped wurtzite GaN up to $10^{20} \mathrm{~cm}^{-3}$. Phys. Rev. B 2014, 90, 075203. [CrossRef] 
22. Pimputkar, S.; Suihkonen, S.; Imade, M.; Mori, Y.; Speck, J.S.; Nakamura, S. Free electroncon centration dependent sub-band gap optical absorption characterization of bulk GaN crystals. J. Cryst. Growth 2015, 432, 49-53. [CrossRef]

23. Vurgaftman, I.; Meyer, J.R. Band parameters for nitrogen-containing semiconductors. J Appl. Phys. 2003, 94, 3675. [CrossRef]

24. Amano, H. Nobel Lecture: Growth of GaN on sapphire via low-temperature deposited buffer layer and realization of p-type GaN by Mg doping followed by low-energy electron beam irradiation. Rev. Mod. Phys. 2015, 87, 1133. [CrossRef]

25. Niebuhr, R.; Bachem, K.H.; Kaufmann, U.; Maier, M.; Merz, C.; Santic, B.; Schlotter, P.; Jurgensen, H. Electrical and optical properties of oxygen doped GaN grown by MOCVD using $\mathrm{N}_{2}$ O. J. Electron. Mater. 1997, 26, 1128-1130. [CrossRef]

26. Zolper, J.C.; Wilson, R.G.; Pearton, S.J.; Stall, R.A. Ca and O ion implantation doping of GaN. Appl. Phys. Lett. 1996, 68, 1945-1947. [CrossRef]

27. Götz, W.; Johnson, N.M.; Chen, C.; Liu, H.; Kuo, C.; Imler, W. Activation energies of Si donors in GaN. Appl. Phys. Lett. 1996, 68, 3144-3146. [CrossRef]

(C) 2017 by the authors. Licensee MDPI, Basel, Switzerland. This article is an open access article distributed under the terms and conditions of the Creative Commons Attribution (CC BY) license (http:/ / creativecommons.org/licenses/by/4.0/). 